\title{
Perioperative Alcohol Withdrawal Syndrome: Care Based on Empathy plus Prescriptions Based on Physiology
}

\author{
Alain Braillon \\ Previously senior consultant, Amiens, France
}

\section{Dear Editor}

The review by Ungur et al. [1] that focused on the perioperative management of alcohol withdrawal syndrome deserves comment.

Firstly, the prevention of delirium is about care [2]. Sadly, poor organization with limited staff availability or education may be the main avoidable causes of delirium [3]. Do we help to reorient the patient to time, date, and place? Do we avoid an unnecessary change of wards? Do we take time to provide reassurance and rehydrate per os?

Secondly, the identification and treatment of preexisting concomitant medical problems is far from limited to alcohol (other examples are pain, infection, etc.). Regarding alcohol users, smoking is very frequently associated with alcohol consumption, and all smokers should benefit from a prescription for nicotine replacement therapy when hospitalized [4]. Ventilated critically ill smokers require supplemental sedatives, neuroleptics, and physical restraint [5].

Thirdly, prevention of thiamine deficiency, an issue too frequently bypassed, is not only about Wernicke-Korsakoff syndrome but also about left ventricular systolic function. It should be systematic for alcohol users who are malnourished or have decompensated liver disease, or when parenteral glucose serum is prescribed. Furthermore, a variety of drugs have been associated with thiamine deficiency including furosemide and antibiotics. Dose estimates are based on data from uncontrolled trials and empirical clinical practice, but $250 \mathrm{mg}$ per day seems to be the minimum recommended thiamine dose $[6,7]$. As its half-life is short, it should be administered 2 or 3 times daily, intravenously or intramuscularly for the first 3-5 days as absorption can be impaired. Thiamine is a coenzyme to various intramitochondrial enzymes involved in carbohydrate metabolism also requiring magnesium. Accordingly, hypomagnesaemia must be corrected for effectiveness. Furthermore, as thiamine is not covered by a Medicare prescription drug plan in the USA, as it is in France by the mandatory healthcare scheme, thiamine deficiency frequently occurs in the ambulatory setting as many patients cannot afford it or don't understand that it could be effective.

Lastly, could Ungur et al. [1] justify their rationale for the following claim: "writing down alcohol-related diagnoses in the medical records requires the patient's presumed consent after shared decision-making"? Is keeping the medical records secret an unsettled issue in Germany? Censoring the most critical data which indicate that a patient requires specific care can only worsen clinical outcomes. Do Ungur et al. [1] require patients' consent for writing down that they are tobacco users?

\section{Conflict of Interest Statement}

There were no conflicts of interest. 


\section{References}

1 Ungur AL, Neumann T, Borchers F, Spies C. Perioperative Management of Alcohol Withdrawal Syndrome. Visc Med. 2020 Jun;36(3): 160-6.

2 Braillon A. Delirium in patients with advanced cancer: screening to cure or caring to prevent? Cancer. 2017 Feb;123(4):704.

3 O’Mahony R, Murthy L, Akunne A, et al. Synopsis of the National Institute for Health and Clinical Excellence guideline for prevention of delirium. Ann Intern Med. 2011 Jun 154(11):746-51.
4 Braillon A. Management of alcohol withdrawal and nicotine replacement therapy. Am J Emerg Med. 2017 Dec;35(12):1956.

5 Lucidarme O, Seguin A, Daubin C, Ramakers M, Terzi N, Beck P, et al. Nicotine withdrawal and agitation in ventilated critically ill patients. Crit Care. 2010;14(2):R58.

6 Thomson AD, Cook CC, Touquet R, Henry JA; Royal College of Physicians, London. The Royal College of Physicians report on alcohol: guidelines for managing Wernicke's encephalopathy in the accident and Emergency Department. Alcohol Alcohol. 2002;37(6):513-21.
7 National Institute for Health and Care Excellence. [Internet] Alcohol-use disorders: diagnosis, assessment and management of harmful drinking (high-risk drinking) and alcohol dependence. Clinical guideline [CG115] 23 February 2011 [cited 20 August 2020]. Available from: https://www.nice.org.uk/guidance/cg115 